\title{
Publisher Correction: Cytological, physiological, and transcriptomic analyses of golden leaf coloration in Ginkgo biloba L
}

Wei-xing Li', Shun-bo Yang ${ }^{1}$, Zhaogeng Lu', Zhi-chong He${ }^{1}$, Yun-ling Ye', Bei-bei Zhao ${ }^{2}$, Li Wang ${ }^{1}$ and Biao Jin (1)

Correction to: Horticulture Research (2018) 5: 12; https://doi.org/10.1038/s41438-018-0015-4; Article published online 1 March 2018.
References

1. Wei-xing Li et al. Cytological, physiological, and transcriptomic analyses of golden leaf coloration in Ginkgo biloba L. Hortic Res 5, 12 https://doi.org/ 10.1038/s41438-018-0015-4 (2018).

In the original publication of this article [1] not all authors were listed correctly. In this correction article the full author list is given. The original publication has been corrected. 